\title{
VAI PARA ONDE? \\ $O$ destino da Ciência Latino-Americana e Caribenha
}

\author{
GOES TO WHERE? \\ The destination of Latin American and Caribbean Science
}

Patricia da Silva Neubert ${ }^{1}$ Rosângela Schwarz Rodrigues ${ }^{2}$

Rogério Mugnaini ${ }^{3}$

\begin{abstract}
RESUMO
A limitada presença dos periódicos de países periféricos entre os títulos indexados em bases internacionais e a publicação de trabalhos, por cientistas destes países, em títulos de núcleo em busca de maior visibilidade expõem a relevância de análises acerca do destino da produção científica periférica indexada em bases de dados internacionais. Esta pesquisa estuda os trabalhos publicados por pesquisadores de países considerados periféricos, neste caso o conjunto de países da América Latina e Caribe, em um universo composto pelo que é denominado ciência global, os periódicos indexados em bases internacionais de renome, aqui representada pela Web of Science. $\mathrm{O}$ objetivo da pesquisa é analisar a dispersão geográfica da produção científica dos países latino-americanos e caribenhos indexada na base. $O$ universo da pesquisa é composto por 251.312 artigos com vínculo de autoria em instituições de países latino-americanos e caribenhos indexados na Web of Science entre 2014 e 2016. Quanto ao destino da produção científica, 75,77\% são publicados por periódicos editados em países externos a América Latina e Caribe, a maioria em países europeus e norte-americanos. Permanecem publicados em títulos editados na região apenas $24,23 \%$ dos artigos. Estes títulos concentram a maioria dos artigos dos campos de Artes \& Humanidades, e Ciências sociais. Em Ciências da Vida \& Biomedicina, publicam pouco mais de um quarto dos artigos, e em Tecnologia e Ciências Físicas menos de 10\% dos trabalhos.
\end{abstract}

Palavras-chave: Comunicação científica. Geografia da ciência. Destinos da produção científica. América Latina e Caribe.

\begin{abstract}
The limited presence of journals from peripheral countries among the titles indexed on international bases and the publication of works by scientists from these countries in core titles are relevant to analyzes scientific production indexed in international databases. This research studies the articles published by researchers from Latin America and the Caribbean, in a universe representative from the global science, the journals indexed in internationally renowned databases, represented by the Web of Science. The objective is to analyze the geographical dispersion of scientific production in Latin American and Caribbean countries indexed in the base. The method is quantitative and collects data in WoS from 2014, 2015, and 2016. The research universe is 251,312 articles linked to authorship in institutions in Latin American and Caribbean countries. The destination of $75.77 \%$ of scientific production is journals with publishers in Europe and North America. Only $24.23 \%$ of the articles remain in titles published in the region. There is a remarkable difference among the countries and the knowledge areas; only Brazil, Colombia, Venezuela, and Cuba have more than $20 \%$ of the articles published in their titles with a media of $15 \%$ in the region. Arts \& Humanities and Social Sciences are the areas with more articles published in their countries while Technology and Physical Sciences published more than $90 \%$ in other countries' titles.

Keywords: Scientific communication. Geography of science. Destinations of scientific production. Latin America and the Caribbean.
\end{abstract}

1 Pesquisadora Membro do Grupo de Pesquisa InformaçãoCientífica: métricas e publicações certificado pela Universidade Federal de Santa Catarina, Brasil. ORCID: http://orcid.org/0000-0002-8909-1898. E-mail: patyneubert@hotmail.com

2 Docente no Programa de Pós-Graduação em Ciência da Informação. Universidade Federal de Santa Catarina, Brasil. ORCID https://orcid.org/0000-0002-9639-6390.E-mail: rosangela.rodrigues@ufsc.br

3 Docente no Programa de Pós-Graduação em Ciência da Informação da Escola de Comunicação e Artes da Universidade de São Paulo, Brasil. ORCID http://orcid.org/0000-0001-9334-3448. E-mail: mugnaini@usp.br 


\section{INTRODUÇÃO}

A publicação científica de países chamados periféricos em periódicos indexados em bases de dados internacionais reconhecidas desperta discussões acerca do movimento de migração dessas publicações. Atraídos pelo maior impacto e visibilidade das pesquisas e motivados pela obtenção de melhores avaliações de sua produção, potencializado pela publicação nos títulos indexados - geralmente não editados em regiões periféricas - os pesquisadores dão origem a uma espécie de movimento migratório de suas publicações.

Isso ocorre principalmente por duas questões históricas associadas à institucionalização de veículos de comunicação científica nas regiões periféricas: 1) as dificuldades de criação e edição de periódicos longe dos centros consolidados (GIBBS, 1995; MIGUEL, 2011; TERRA-FIGARI, 2008), parcialmente solucionada pelas tecnologias e pelo movimento de acesso aberto (OA) (ALONSO GAMBOA; SÁNCHEZ ISLAS, 2005; GUÉDON, 2010; TERRA-FIGARI, 2008) e, 2) pela dificuldade de indexação destes títulos (quando existem) em bases de dados internacionais, essencial para a contabilidade das citações, logo de prestígio (MIGUEL, 2011; TERRA-FIGARI, 2008).

A ciência considerada mainstream está associada ao prestígio conferido pela indexação e alicerçado nos indicadores e rankings de periódicos que as bases de dados publicam e, das quais a Web of Science (WoS) e o Fator Impacto (FI) tem exercido papel central há décadas (BEIGEL, 2014, 2016; GUÉDON, 2001, 2010, 2011). Tradicionalmente, é a produção veiculada em periódicos indexados em bases de dados internacionais e multidisciplinares, e publicados majoritariamente por editores comerciais de nacionalidades específicas - EUA, Reino Unido e Países Baixos, e que revela os limites da geopolítica das bases de dados ditas internacionais. (BEIGEL, 2014, 2016; GUÉDON, 2001, 2010, 2011; LARIVIÉRE; HAUSTEIN; MONGEON, 2015; PACKER 2011).

Nesse cenário, pesquisadores de nações fora dos centros tendem a submeter seus trabalhos entre os títulos considerados mainstream em busca de maior visibilidade, desprivilegiando os periódicos que não compõem esse grupo - ainda que de qualidade (TERRA-FíGARI, 2008; STREHL et al., 2016). Um padrão comportamental reproduzido por outros pesquisadores, frequentemente estimuladospor instrumentos de avaliação da produção científica e, que tende a diminuir as chances dos títulos periféricos de alcançarem uma posição relevante - à medida que os trabalhos considerados de maior qualidade e potencial de citação são sistemática e continuamente submetidos aos títulos mainstream, concentrados nos países sede das principais grandes editoras comerciais. 
As análises globais do desenvolvimento da Ciência tendem a refletir uma geografia específica, seja na indexação em bases de dados, na editoria dos periódicos, no volume da produção científica esua citação, em rankings universitários ou mesmo nos locais de publicação do conhecimento científico, que tendem a refletir o que é considerado como o centro da publicação científica global (BORNMANN; WAGNER; LEYDESDORFF, 2018; CSOMÓS, 2018; JÖNS; HOYLER, 2013; MAISONOBE et al., 2017; PAASI, 2005). No entanto, é necessário destacar que a ciência mainstream ignora parte da produção científica mundial, não sendo representativa da maioria das nações. Quando se fala em internacionalização, sobretudo ao se referir à publicação em títulos mainstream, está-se fazendo referência às geografias específicas (PAASI, 2005), à geopolítica das bases de dados, de origem predominantemente angloamericana e comercial.

Quanto à presença de localidades periféricas em análises científicas globais, são os países asiáticos que tendem a se destacar, embora mesmo ao apresentarem índices de produção científica semelhantes aos países com alto desenvolvimento científico, seus periódicos ainda são considerados como locais ou regionais (BORNMANN; WAGNER; LEYDESDORFF, 2018; CSOMÓS, 2018; MAISONOBE et al., 2017). A diferença de status pode ser atribuída à endogenia nos periódicos editados nessas regiões que possuem dificuldade de atrair autores de outros países, mesmo publicando em inglês (STREHL et al., 2016). Ainda assim, os critérios para que um título seja considerado internacional ainda são vagos e demandam discussão.

As discussões acerca da evasão da produção científica centram-se na relação com o acúmulo de capital científico, associado ao prestígio e a visibilidade obtidos com a publicação em periódicos mainstream. Entretanto, suas implicações ecoam na institucionalização e organização dos campos científicos, especialmente relacionados a capacidade editorial científica, na criação e constituição de mecanismos para comunicar a Ciência produzida nas regiões periféricas.

Assim, as análises da relação entre o local de produção e de publicação do conhecimento científico estão associadas ao grau de autonomia ou de independência dos campos científicos (JAGODZINSKI-SIGOGNEAU; COURTIAL; LATOUR, 1982), neste caso especialmente associados ao sistema global de comunicação da Ciência.

A limitada presença dos periódicos de países periféricos entre os títulos indexados em bases internacionais e a publicação de trabalhos, por cientistas destes países, em títulos mainstream em busca da validação científica global e das consequentes visibilidade e citações, expõem a relevância de análises acerca do destino da produção científica periférica indexada em bases de dados internacionais. 
Este estudo analisa a dispersão geográfica da produção científica de pesquisadores da América Latina e Caribe, no contexto da ciência global, aqui representada pelo universo delineado pela base WoS.

\section{PROCEDIMENTOS METODOLÓGICOS}

Os dados foram coletados a partir da busca pelos países latino-americanos e caribenhos, no campo país/região (CU), na Coleção Principal da WoS, no período de 2014 a 2016, resultando no universo da pesquisa, composto por 251.312 artigos.

A análise da dispersão geográfica da produção científica, considera a nacionalidade da origem da produção científica - baseada no endereço institucional dos autores e limitada aos países latinoamericanos e caribenhos -, e do destino da publicação - pela nacionalidade dos editores. Esse dado foi retirado do endereço atribuído a cada editor (campo PI), vinculado individualmente a cada artigo - a despeito do caráter multinacional de inúmeros grupos editoriais e da dificuldade em identificar sua atuação, uma das limitações deste estudo - considerando assim a informação registrada na base de dados.

A dispersão da produção científica foi medida pelas taxas de evasão e de permanência dos artigos, calculadas considerando, respectivamente, o total de artigos vinculados ao país ou região, e 0 montante desses artigos que foram publicados em títulos editados: no mesmo país ou região, no caso da taxa de permanência; ou num país ou região diferente, para a taxa de evasão, conforme o Quadro 1:

Quadro 1 - Fórmula para cálculo da Taxa de permanência e evasão

\begin{tabular}{|c|}
\hline Taxa de permanência \\
\hline$\frac{\text { total artigos vinculados ao país/região publicados em periódicos editados no país/região }}{\text { total artigos vinculados ao país/região }} \times 100$ \\
\hline Taxa de evasão \\
\hline total artigos vinculados ao país/região publicados em periódicos editados no exterior \\
total artigos vinculados ao país/região
\end{tabular}

Fonte: Neubert e Rodrigues (2018) 


\section{RESULTADOS E DISCUSSÃO}

A produção científica latino-americana e caribenha indexada na WoS no período analisado é composta por 251.312 artigos, sendo o Brasil o país com maior participação na autoria das publicações, seguido pelo México, Argentina, Chile e Colômbia. Observa-se a proeminente representação da produção científica brasileira entre os títulos indexados na WoS, sendo a participação nacional mais elevada em todos os campos do conhecimento (Tabela um).

Embora predomine entre os artigos de cada campo do conhecimento, é em Ciências da Vida \& Biomedicina que se evidencia a maior participação da produção científica brasileira em relação à região (sendo $59,27 \%$ do referido campo e $33,76 \%$ do total de artigos), e ainda, $62,23 \%$ de sua produção doméstica. Este campo contempla, entre seus subcampos, as Ciências agrárias, reconhecidamente uma especialidade científica brasileira (PEDROSA; CHAIMOVICH, 2015; SOETE et al., 2015; UNITED NATIONS EDUCATIONAL, SICENTIFIC AND CULTURAL ORGANIZATION, 2015; SANTIN, CAREGNATO, 2019).

Tabela 1 - Produção científica latino-americana e caribenha segundo país de origem e campo do conhecimento (WoS/2014-2016)

\begin{tabular}{|c|c|c|c|c|c|c|c|c|c|c|c|c|}
\hline \multirow[t]{2}{*}{ Origem } & \multicolumn{2}{|c|}{ Total } & \multicolumn{2}{|c|}{$\begin{array}{l}\text { Ciências da Vida } \\
\text { \& Biomedicina }\end{array}$} & \multicolumn{2}{|c|}{ Ciências Físicas } & \multicolumn{2}{|c|}{ Tecnologia } & \multicolumn{2}{|c|}{ Ciências Sociais } & \multicolumn{2}{|c|}{$\begin{array}{c}\text { Artes \& } \\
\text { Humanidades }\end{array}$} \\
\hline & N & $\%$ & N & $\%$ & N & $\%$ & N & $\%$ & N & $\%$ & $\mathrm{~N}$ & $\%$ \\
\hline BR & 136.321 & 54,2 & 84.840 & 59,3 & 27.817 & 48,3 & 26.141 & 53,1 & 9.468 & 39,7 & 3.232 & 42,4 \\
\hline MX & 42.100 & 16,7 & 21.501 & 15,0 & 12.341 & 21,4 & 10.081 & 20,5 & 3.574 & 15,0 & 877 & 11,5 \\
\hline AR & 28.384 & 11,3 & 16.035 & 11,2 & 7.393 & 12,8 & 4.693 & 9,5 & 2.682 & 11,3 & 1.359 & 17,9 \\
\hline $\mathrm{CL}$ & 24.480 & 9,7 & 10.717 & 7,5 & 8.200 & 14,2 & 4.170 & 8,5 & 3.013 & 12,6 & 1.302 & 17,1 \\
\hline $\mathrm{CO}$ & 14.106 & 5,6 & 6.487 & 4,5 & 2.894 & 5,0 & 3.194 & 6,5 & 2.631 & 11,0 & 492 & 6,5 \\
\hline PE & 3.631 & 1,4 & 2.351 & 1,6 & 556 & 0,9 & 553 & 1,1 & 451 & 1,9 & 69 & 0,9 \\
\hline $\mathrm{CU}$ & 3.628 & 1,4 & 1.729 & 1,2 & 687 & 1,2 & 751 & 1,5 & 805 & 3,4 & 51 & 0,7 \\
\hline UY & 3.473 & 1,4 & 2.259 & 1,6 & 686 & 1,2 & 501 & 1,0 & 368 & 1,5 & 60 & 0,8 \\
\hline EC & 3.458 & 1,4 & 1.697 & 1,2 & 575 & 1,0 & 729 & 1,5 & 742 & 3,1 & 92 & 1,2 \\
\hline VE & 3.439 & 1,4 & 1.731 & 1,2 & 751 & 1,3 & 610 & 1,2 & 504 & 2,1 & 105 & 1,4 \\
\hline CR & 2.123 & 0,8 & 1.270 & 0,9 & 263 & 0,5 & 334 & 0,7 & 367 & 1,5 & 58 & 0,8 \\
\hline PA & 1.240 & 0,5 & 970 & 0,7 & 116 & 0,2 & 201 & 0,4 & 41 & 0,2 & 5 & 0,1 \\
\hline BO & 847 & 0,3 & 601 & 0,4 & 154 & 0,3 & 117 & 0,2 & 69 & 0,3 & 14 & 0,2 \\
\hline GT & 519 & 0,2 & 394 & 0,3 & 19 & 0,0 & 64 & 0,1 & 81 & 0,3 & 4 & 0,1 \\
\hline PY & 446 & 0,2 & 343 & 0,2 & 37 & 0,1 & 58 & 0,1 & 35 & 0,2 & 2 & 0,1 \\
\hline $\mathrm{NI}$ & 289 & 0,1 & 173 & 0,1 & 25 & 0,1 & 24 & 0,1 & 76 & 0,3 & 1 & 0,1 \\
\hline DO & 284 & 0,1 & 214 & 0,1 & 26 & 0,1 & 22 & 0,1 & 37 & 0,2 & 3 & 0,1 \\
\hline HT & 255 & 0,1 & 210 & 0,1 & 20 & 0,1 & 18 & 0,1 & 24 & 0,1 & 2 & 0,1 \\
\hline PR & 223 & 0,1 & 156 & 0,1 & 40 & 0,1 & 41 & 0,1 & 3 & 0,1 & - & - \\
\hline SV & 214 & 0,1 & 140 & 0,1 & 19 & 0,1 & 24 & 0,1 & 35 & 0,2 & 13 & 0,2 \\
\hline $\mathrm{HN}$ & 183 & 0,1 & 145 & 0,1 & 12 & 0,1 & 21 & 0,1 & 14 & 0,1 & 2 & 0,1 \\
\hline ALeC & 251.312 & 100 & 143.135 & 100 & 57.604 & 100 & 49.276 & 100 & 23.846 & 100 & 7.611 & 100 \\
\hline
\end{tabular}

Fonte: dados da pesquisa (2017).

Legenda: BR (Brasil), MX (México), AR (Argentina), CL (Chile) CO (Colômbia). PE (Peru), CU (Cuba), UY (Uruguai), EC (Equador), VE (Venezuela, CR (Costa Rica), PA (Panamá), BO (Bolívia), GT (Guatemala), PY (Paraguai), NI (Nicarágua), DO (República Dominicana), HT (Haiti), PR (Porto Rico), SV (El Salvador), HN (Honduras), ALeC (América Latina e Caribe). Nota: Percentuais arredondados em relação ao total de artigos em cada campo do conhecimento. 


\subsection{Dispersão da produção científica}

Os títulos editados na América Latina e Caribe são o destino de publicação de $24,23 \%$ dos artigos produzidos na região, podendo-se observar variação em relação aos países destino dos artigos, sendo o Brasil o principal, com 15,21\%. Os totais de linha revelam que Venezuela, Cuba, Colômbia, Brasil, Costa Rica, Paraguai e Equador - em ordem decrescente, sendo os que destinam pelo menos um quinto de seus artigos para periódicos publicados por editoras de seus países.

Ao se analisar a taxa permanência nacional (Tabela 2), observa-se a Venezuela com a maior taxa (26,81\%), seguida do Brasil (26,6\%), Cuba (23,95\%) e Colômbia (20,91\%), sendo os que destinam um quinto de sua produção a periódicos editados no país.

Tabela 2 - Percentual de permanência da produção científica latino-americana e caribenha segundo país de origem e destino na região (WoS/2014-2016)

\begin{tabular}{|c|c|c|c|c|c|c|c|c|c|c|c|c|c|c|c|c|}
\hline \multirow{2}{*}{ Autor } & \multicolumn{15}{|c|}{ País da revista } & \multirow{2}{*}{ Total } \\
\hline & BR & MX & CL & $\mathrm{co}$ & $A R$ & VE & $\mathrm{CU}$ & CR & $\mathrm{EC}$ & PE & UY & PY & NI & BO & sv & \\
\hline AL & 15,2 & 2,2 & 2,1 & 1,8 & 1,1 & 0,6 & 0,4 & 0,4 & 0,2 & 0,1 & 0,1 & 0,1 & 0,1 & 0,1 & 0,1 & 24,2 \\
\hline BR & 26,6 & 0,1 & 0,4 & 0,3 & 0,2 & 0,1 & 0,1 & 0,1 & 0,1 & 0,1 & 0,1 & 0,0 & - & - & - & 27,9 \\
\hline MX & 1,4 & 10,9 & 1,3 & 1,1 & 0,5 & 0,7 & 0,1 & 0,5 & 0,1 & 0,1 & 0,1 & 0,1 & 0,0 & 0,1 & - & 16,6 \\
\hline $\mathrm{CL}$ & 1,5 & 0,8 & 13,1 & 1,2 & 0,6 & 0,7 & 0,1 & 0,1 & 0,1 & 0,1 & 0,1 & 0,1 & - & 0,1 & - & 18,1 \\
\hline $\mathrm{CO}$ & 2,7 & 1,5 & 1,5 & 20,9 & 0,6 & 0,7 & 0,1 & 0,7 & 0,1 & 0,1 & 0,1 & 0,1 & 0,1 & - & 0,0 & 29,0 \\
\hline$A R$ & 2,7 & 1,0 & 2,1 & 1,3 & 7,2 & 0,2 & 0,1 & 0,2 & 0,1 & 0,1 & 0,1 & - & 0,1 & 0,1 & - & 15,1 \\
\hline VE & 2,0 & 1,7 & 1,6 & 3,9 & 0,7 & 26,8 & 0,2 & 1,2 & 0,3 & 0,2 & 0,1 & 0,1 & 0,1 & - & - & 38,9 \\
\hline $\mathrm{CU}$ & 2,4 & 4,2 & 1,5 & 1,7 & 0,4 & 0,3 & 23,9 & 0,5 & 1,6 & 0,1 & 0,1 & - & - & - & 0,1 & 36,8 \\
\hline $\mathrm{CR}$ & 1,5 & 1,2 & 1,3 & 1,1 & 0,5 & 0,5 & 0,1 & 18,9 & 0,1 & 0,1 & 0,1 & - & - & - & - & 25,3 \\
\hline EC & 2,0 & 1,3 & 1,3 & 2,0 & 0,7 & 1,0 & 4,8 & 0,5 & 9,2 & 0,3 & 0,1 & - & - & - & - & 23,2 \\
\hline PE & 1,8 & 1,0 & 2,1 & 1,1 & 0,7 & 0,4 & 0,1 & 0,2 & 0,1 & 6,7 & 0,1 & 0,1 & - & - & 0,1 & 14,5 \\
\hline UY & 5,6 & 0,8 & 2,5 & 0,4 & 1,2 & 0,3 & 0,1 & 0,1 & - & 0,1 & 3,5 & - & - & 0,1 & - & 14,7 \\
\hline PY & 6,5 & 0,5 & 3,1 & 0,7 & 1,8 & - & 0,2 & - & - & - & 0,2 & 11,9 & - & - & - & 24,9 \\
\hline $\mathrm{NI}$ & 0,3 & 0,7 & 0,7 & - & 0,7 & 1,7 & 0,3 & 2,1 & - & - & - & - & 12,1 & - & - & 18,7 \\
\hline BO & 2,1 & 0,6 & 1,1 & 0,9 & 1,3 & 0,5 & 0,3 & 0,7 & 0,5 & - & - & - & - & 0,7 & - & 8,7 \\
\hline SV & 1,4 & 2,3 & 0,9 & 1,4 & 0,5 & - & 0,9 & 1,4 & - & - & 0,5 & 0,5 & - & - & 5,1 & 14,9 \\
\hline DO & 3,2 & 1,4 & 1,4 & - & 1,1 & 0,7 & 0,7 & 0,3 & - & - & - & - & - & - & - & 8,8 \\
\hline GT & 0,9 & 2,3 & 0,4 & 0,6 & 0,4 & 0,8 & - & 0,4 & 0,4 & - & - & - & - & - & - & 6,2 \\
\hline $\mathrm{HN}$ & 1,1 & 2,7 & 1,6 & 0,5 & - & - & 0,5 & 0,5 & - & - & - & - & - & - & - & 7,1 \\
\hline HT & 0,4 & - & - & 0,4 & - & - & - & - & - & - & - & - & - & - & - & 0,8 \\
\hline PA & 0,7 & 0,6 & 0,3 & 0,6 & 0,3 & 0,9 & 0,2 & 0,2 & 0,00 & - & - & 0,1 & - & - & - & 3,9 \\
\hline PR & 3,6 & 1,8 & 0,5 & 1,3 & - & - & - & 1,4 & - & - & - & 0,4 & - & - & - & 8,9 \\
\hline
\end{tabular}

Fonte: dados da pesquisa.

Legenda: BR (Brasil), MX (México), AR (Argentina), CL (Chile) CO (Colômbia). PE (Peru), CU (Cuba), UY (Uruguai), EC (Equador), VE (Venezuela, CR (Costa Rica), PA (Panamá), BO (Bolívia), GT (Guatemala), PY (Paraguai), NI (Nicarágua), DO (República Dominicana), HT (Haiti), PR (Porto Rico), SV (El Salvador), HN (Honduras), AL (América Latina e Caribe). Nota: As linhas expressam o percentual arredondado de artigos originados em cada país, e respectivos países de destino. 
As colunas da Tabela 2 mostram que o Brasil é o país cujos periódicos são mais utilizados como destino dos demais países da região, com destaque para o Paraguai $(6,50 \%)$ e Uruguai $(5,61 \%)$, além de diversos outros com pelo menos dois \% de seus artigos. México e Chile são destino de quatro outros países, sendo o primeiro procurado por países da América Central, enquanto que o segundo da América do Sul. Já a Colômbia é destino mais frequente de Venezuela (3,95\%) e Equador (2,00\%). Efinalmente Cuba e Costa Rica se destacam por serem mais procurados, respectivamente, por Equador $(4,86 \%)$ e Nicarágua $(2,08 \%)$.

A análise do destino da produção científica da região, mostra que $75,77 \%$ dos artigos são publicados por periódicos editados fora da América Latina e Caribe. Este feito aponta para a aceitação e universalização da Ciência produzida na região e, uma vez que a comunicação de grande parte das pesquisas desses países é publicada nos títulos de maior prestígio mundial, muitos com alto Fator de Impacto. Tal alcance significa que a ciência produzida na região foi capaz de passar pelos rigorosos filtros de qualidade alardeados pelos títulos mainstream. Assim, permanecem publicados em títulos editados na América Latina e Caribe os remanescentes 24,23\%. Confrontando as taxas de permanência e evasão, pode-se observar entre os países da Figura um que as maiores taxas de permanência da produção científica foram identificadas nos campos de Artes \& Humanidades e Ciências Sociais. Especialmente El Salvador (84,6\%), Brasil $(77,0 \%)$, Colômbia $(55,5 \%)$ e Chile $(51,62 \%)$ publicam mais do que a metade de sua produção científica em periódicos da região, sendo os três últimos os maiores produtores do campo (conforme Tabela um). Colômbia $(54,0 \%)$ e Brasil $(52,0 \%)$ ainda são destacados pela mesma razão, quando publicando em Ciências Sociais, além de outros quatro países: Paraguai $(57,14 \%)$, Cuba $(57,1 \%)$ e Venezuela $(54,0 \%)$. Desses países, Brasil e Colômbia se destacam por um maior percentual de publicação em títulos nacionais, enquanto os demais países recorrem mais aos outros países da região (conforme observado na Tabela dois). Finalmente, Argentina e México apresentam uma taxa de permanência mais pronunciada em Ciências Sociais, seguido de Artes \& Humanidades, ainda que em menor magnitude que os anteriores.

As taxas de permanência evidenciam o esforço de publicação em títulos mainstream, e ao mesmo tempo, o resultado de significativos esforços de institucionalização e internacionalização de seus periódicos, que significam reconhecimento e visibilidade internacional dos títulos editados por cada país.

É importante observar que Brasil Colômbia e Chile apresentam maior produção, tanto na WoS como no SciELO Citation Index, principalmente nas áreas de ciências humanas e sociais o SciELO 
fica mais evidente (LUCIO-ARIA; VELEZ-CUARTAS; LEYDESDORFF, 2015). Além disso, o peso das publicações em Acesso Aberto é mais destacado entre os países do Cone Sul em ambas as bases, e para os países andinos no SciELO (MINNITI; SANTORO; BELLI, 2018). Isso nos leva à constatação de que 0 desenvolvimento de infraestrutura de comunicação científica nesses países avançou significativamente, sendo que os mesmos são os mais volumosos em número de artigos, entre todos os que compunham a Rede SciELO em 2014 (PACKER; COP; SANTOS, 2014). A maior representatividade das sociais e humanas em fontes nacionais pode ser resultado da menor cobertura dessas áreas na WoS.

No outro extremo estão os países cuja taxa de evasão atinge aproximadamente $100 \%$ de sua produção (Bolívia, Guatemala, Haiti, Honduras, Nicarágua, Panamá, Peru, Porto Rico, República Dominicana e Uruguai). Alguns desses, quando publicam em periódicos da região, o fazem majoritariamente nas Ciências Sociais ou Artes \& Humanidades, e representam pequenos percentuais da produção total.

Cabe notar a diversidade de desempenho dos vários países que compõe a América Latina e Caribe, tanto na produção de artigos como na publicação de periódicos científicos indexados na WoS, mostrando uma heterogeneidade que não permite uma análise sem o detalhamento dos diversos países que a compõe.

A figura 1 mostra ainda as diferenças das várias áreas do conhecimento, cujo comportamento varia muito e dificulta uma análise mesmo dentro de cada país. Prescindir do detalhamento das áreas do conhecimento mascara o desempenho e ignora as especificidades de cada uma. Esta situação indica que as taxas de evasão na região devem ser calculadas por país e por área do conhecimento. 
Figura 1 - Dispersão da produção científica latino-americana e caribenha por país de origem, destino (no país, na ALaC ou outros países) e campo do conhecimento (WoS/2014-2016)

Ciências da Vida \& Biomedicina
Ciências Físicas
Tecnologia
Ciências Sociais
Artes \& Humanidades

Ciências da Vida \& Biomedicina Ciências Físicas

Tecnologia

Ciências Sociais

Artes \& Humanidades

Ciências da Vida \& Biomedicina

Ciências Físicas

Tecnologia

Ciências Sociais

Artes \& Humanidades

Ciências da Vida \& Biomedicina

Ciências Físicas

Tecnologia

Ciências Sociais

Artes \& Humanidades

Ciências da Vida \& Biomedicina

Ciências Físicas

Tecnologia

Ciências Sociais

Artes \& Humanidades

Ciências da Vida \& Biomedicina

Ciências Físicas

Tecnologia

Ciências Sociais

Artes \& Humanidades

Ciências da Vida \& Biomedicina

Ciências Físicas

Tecnologia

Ciências Sociais

Artes \& Humanidades

Legenda:

Do país

Da ALaC
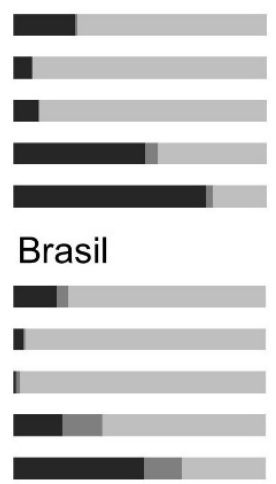

Chile

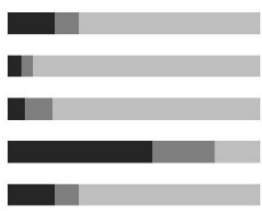

Cuba

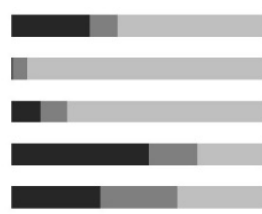

\section{Venezuela}

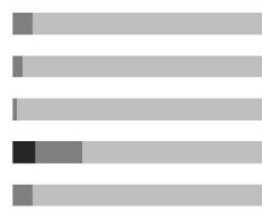

Bolívia

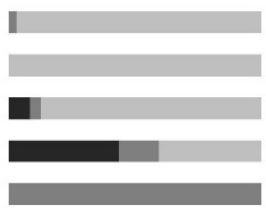

\section{Nicarágua}

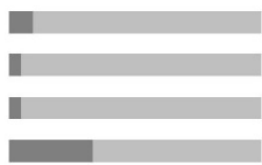

Porto Rico
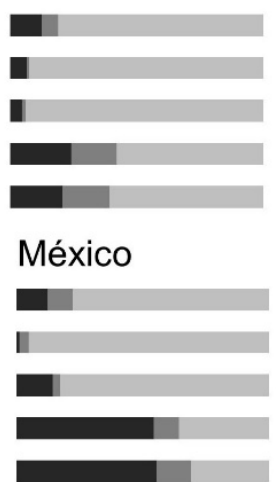

\section{Colômbia}

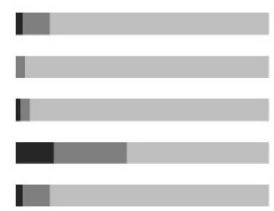

Uruguai

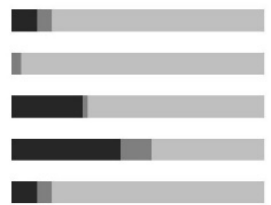

\section{Costa Rica}

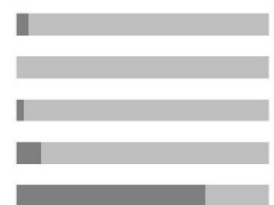

\section{Guatemala}

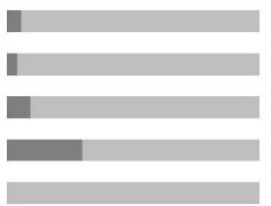

Rep. Dominicana

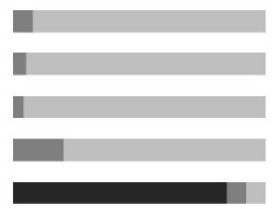

El Salvador

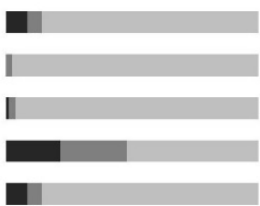

Argentina

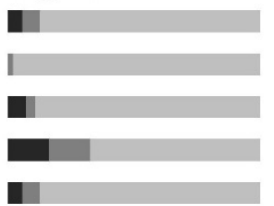

Peru

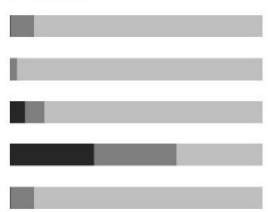

Equador

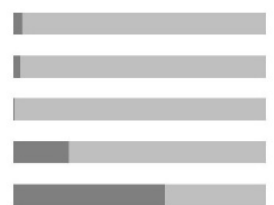

Panamá

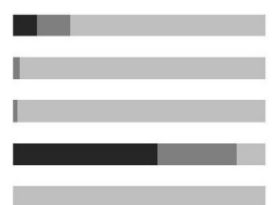

\section{Paraguai}

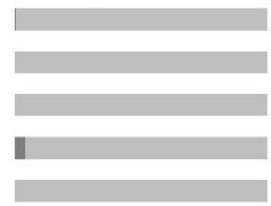

Haiti

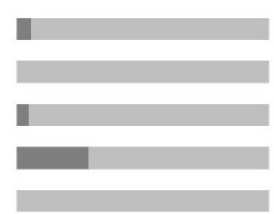

Honduras

Outros países

Fonte: dados da pesquisa. 


\subsection{A evasão da produção científica}

A maioria da produção científica latino-americana e caribenha indexada na WoS é publicada em periódicos editados em países europeus $(41,26 \%)$ e norte-americanos $(31,27 \%)$. Entre os países europeus, o maior destinatário da produção científica regional é a Inglaterra, que recebe $18,35 \%$ dos artigos, seguida pelos Países Baixos (10,52\%). Esses dois países, mais a Alemanha, estão entre as nacionalidades predominantes na WoS, e publicam $46,6 \%$ dos artigos em Ciências Físicas, 44,44\% em Tecnologia e 29,83\% em Ciências da Vida \& Biomedicina, enquanto em Ciências Sociais e Artes \& Humanidades sua representatividade é consideravelmente inferior, $19,78 \%$ e 7,82\%, respectivamente. No campo de Artes \& Humanidades, entre os países europeus, a Espanha é o destino preferencial $(14,77 \%)$ das publicações (Tabela tres).

$\mathrm{Na}$ América do Norte, a segunda região de maior acúmulo dos artigos latino-americanos e caribenhos (31,37\% do total), os EUA são o destino preferido, em todos os campos do conhecimento, sendo o país que recebe maior volume de artigos dos campos de Tecnologia, Ciências Físicas e Ciências da Vida \& Biomedicina, 36,58\%, 34,26\% e 31,85\%, respectivamente (Tabela 3). 
Tabela 3 - Destino da produção científica latino-americana e caribenha (continente e país) segundo campo do conhecimento (WoS/2014-2016)

\begin{tabular}{|c|c|c|c|c|c|c|c|c|c|c|c|c|}
\hline Destino por Continente & \multicolumn{2}{|c|}{ Todos os campos } & \multicolumn{2}{|c|}{$\begin{array}{l}\text { Ciências da Vida } \\
\text { \& Biomedicina }\end{array}$} & \multicolumn{2}{|c|}{ Ciências Físicas } & \multicolumn{2}{|c|}{ Tecnologia } & \multicolumn{2}{|c|}{ Ciências Sociais } & \multicolumn{2}{|c|}{$\begin{array}{c}\text { Artes \& } \\
\text { Humanidades }\end{array}$} \\
\hline Europa & 103.684 & 41,26 & 54.324 & 37,95 & 32.478 & 56,38 & 25.795 & 52,35 & 7.389 & 30,99 & 2.061 & 27,08 \\
\hline América Latina e Caribe & 60.898 & 24,23 & 36.789 & 25,70 & 3.704 & 6,43 & 4.482 & 9,10 & 12.502 & 52,43 & 4.849 & 63,71 \\
\hline Ásia & 4.327 & 1,72 & 2.543 & 1,78 & 1.319 & 2,29 & 766 & 1,55 & 49 & 0,21 & 16 & 0,21 \\
\hline Oceania & 3.121 & 1,24 & 3.028 & 2,12 & 96 & 0,17 & 78 & 0,16 & 48 & 0,20 & 6 & 0,08 \\
\hline Ásia / Oceania & 7 & 0,00 & 3 & 0,00 & 2 & 0,00 & 2 & 0,00 & - & - & - & - \\
\hline Ásia / África & 4 & 0,00 & 3 & 0,00 & - & - & 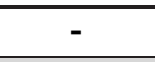 & & - & - & 1 & 0,01 \\
\hline Total & 251.312 & 100 & 143.135 & 100 & 57.604 & 100 & 49.276 & 100 & 23.846 & 100 & 7.611 & 100 \\
\hline Destino por País & \multicolumn{2}{|c|}{ Todos os campos } & \multicolumn{2}{|c|}{$\begin{array}{l}\text { Ciências da Vida } \\
\text { \& Biomedicina }\end{array}$} & \multicolumn{2}{|c|}{ Ciências Físicas } & \multicolumn{2}{|c|}{ Tecnologia } & \multicolumn{2}{|c|}{ Ciências Sociais } & \multicolumn{2}{|c|}{$\begin{array}{c}\text { Artes \& } \\
\text { Humanidades }\end{array}$} \\
\hline Estados Unidos & $\underset{77.990}{\mathbf{N}}$ & $\begin{array}{c}\% \\
31,03\end{array}$ & $\begin{array}{c}\mathbf{N} \\
45.591\end{array}$ & $\begin{array}{c}\% \\
31,85\end{array}$ & $\begin{array}{c}\mathbf{N} \\
19.734\end{array}$ & $\begin{array}{c}\% \\
34,26\end{array}$ & $\begin{array}{c}\mathbf{N} \\
18.025\end{array}$ & $\begin{array}{c}\% \\
36,58\end{array}$ & $\begin{array}{c}\mathbf{N} \\
3.770\end{array}$ & $\begin{array}{c}\% \\
15,81\end{array}$ & $\begin{array}{c}N \\
654\end{array}$ & $\begin{array}{c}\% \\
8,59\end{array}$ \\
\hline Alemanha & 7.561 & 3,01 & 4.353 & 3,04 & 2.827 & 4,91 & 1.029 & 2,09 & 200 & 0,84 & 90 & 1,18 \\
\hline Suíça & 7.319 & 2,91 & 3.223 & 2,25 & 2.514 & 4,36 & 2.657 & 5,39 & 331 & 1,39 & 14 & 0,18 \\
\hline Espanha & 5.816 & 2,31 & 2.232 & 1,56 & 233 & 0,40 & 319 & 0,65 & 2.025 & 8,49 & 1.124 & 14,77 \\
\hline México & 5.538 & 2,20 & 2.970 & 2,07 & 980 & 1,70 & 552 & 1,12 & 1.224 & 5,13 & 214 & 2,81 \\
\hline Chile & 5.162 & 2,05 & 2.992 & 2,09 & 491 & 0,85 & 144 & 0,29 & 1.050 & 4,40 & 922 & 12,11 \\
\hline Colômbia & 4.549 & 1,81 & 1.154 & 0,81 & 42 & 0,07 & 585 & 1,19 & 2.232 & 9,36 & 602 & 7,91 \\
\hline França & 2.888 & 1,15 & 1.057 & 0,74 & 1.653 & 2,87 & 177 & 0,36 & 43 & 0,18 & 78 & 1,02 \\
\hline Argentina & 2.798 & 1,11 & 1.823 & 1,27 & 21 & 0,04 & 111 & 0,23 & 820 & 3,44 & 183 & 2,40 \\
\hline Nova Zelândia & 2.598 & 1,03 & 2.575 & 1,80 & 10 & 0,02 & 58 & 0,12 & 23 & 0,10 & - & - \\
\hline Outros (inferior a 1\%) & 18.338 & 7,31 & 11.342 & 7,93 & 2.959 & 5,15 & 2.022 & 4,1 & 2.443 & 10,25 & 434 & 5,73 \\
\hline Total & 251.312 & 100 & 143.135 & 100 & 57.604 & 100 & 49.276 & 100 & 23.846 & 100 & 7.611 & 100 \\
\hline
\end{tabular}

Fonte: dados da pesquisa (2017).

Nota: Percentual em relação ao total da produção regional em cada campo do conhecimento. 
A identificação da Inglaterra (18,35\%), Países Baixos (10,52\%) e EUA $(31,03 \%)$ como destinos preferenciais da produção científica latino-americana e caribenha (Tabela tres) é coerente com os resultados dos estudos que identificam estes locais como os centros mundiais de produção e editoria científica (CSOMÓS, 2018; LARIVIÈRE; HAUSTEIN; MONGEON, 2015; MAISONOBE et al., 2017; PAASI, 2005).

Os dados mostram que a evasão da produção científica da região pela publicação em títulos editados fora da América Latina e Caribe, é maior nos campos de Ciências Físicas, Tecnologia e Ciências da Vida \& Biomedicina que acumulam as maiores taxas, respectivamente, 93,57\%, 90,90\% e 74,30\% do total de cada campo (Tabela tres), expondo uma elevada dependência de títulos estrangeiros para a publicação, exibindo uma forte dependência do sistema de comunicação científica internacional como uma característica do campo, o que que indica uma limitada capacidade de articulação para inclusão de títulos regionais no cenário global.

Dos apenas 6,43\% dos artigos do campo de Ciências Físicas que permanecem na região, a maioria é publicada em títulos brasileiros (3,68\%) e mexicanos (1,7\%) (Tabela 3). Enquanto a maioria dos países apresentam taxas de evasão de 100\%, Brasil (7,16\%), México (6,51\%) e Cuba (5,24\%) apresentam as maiores taxas de permanência nacional (Figura um), ainda assim bastante reduzidas. São dados que expõem o padrão de comunicação no campo de Ciências Físicas que considera a produção internacionalizada aquela que é publicada em títulos europeus e norte-americanos, sedes das grandes editoras comerciais

No campo de Tecnologia permanecem na região apenas $9,10 \%$ dos trabalhos, a maior parte deste publicados em periódicos brasileiros (5,53\%) (Tabela tres). Países como Bolívia, El Salvador, Guatemala, Haiti, Honduras, Panamá, Paraguai, Porto Rico e República Dominicana tem 100\% de seus artigos publicados em títulos estrangeiros (Figura um). A menor taxa de evasão da produção nacional em Tecnologia é apresentada por Costa Rica, país no qual $71,86 \%$ dos artigos são publicados em títulos estrangeiros.

O campo de Ciências da Vida \& Biomedicina publica em títulos latino-americanos e caribenhos $25,7 \%$ dos artigos, percentual ainda inferior a participação dos destinos europeus e norteamericanos, mas expressivo em comparação a permanência dos artigos na região nos camposde Tecnologia $(9,10 \%)$ e Ciências Físicas (6,43\%) (Tabela 3). Essa divergência entre os campos é resultado da especialização científica nos campos de Ciências da Vida \& Biomedicina na América Latina e Caribe, reiterada continuamente em variados estudos sobre a produção científica mundial (RED DE 
INDICADORES DE CIENCIA Y TECNOLOGÍA, 2019; UNITED NATIONS EDUCATIONAL, SICENTIFIC AND CULTURAL ORGANIZATION, 2015). Além disso, é também o indício do investimento na consolidação de instrumentos de publicação e valorização dos títulos desta especialidade editados na região.

As Ciências da Vida \& Biomedicina também apresentam uma expressiva taxa de evasão da produção latino-americana e caribenha, $74,3 \%$ dos artigos (Tabela tres). 0 que permanece publicado em títulos editados na região é em grande parte publicado no Brasil (18\%) (Tabela 3). A permanência nacional é de 31,14\% na Venezuela, 29,06\% no Brasil, 18,57\% em Cuba, $17,14 \%$ no Chile, $12,44 \%$ na Colômbia, 12,25\% no México, e 10,24\% na Costa Rica (Figura 1). Bolívia, El Salvador, Guatemala, Haiti, Honduras, Nicarágua, Panamá, Porto Rico e República Dominicana tem evasão de 100\% de sua produção.

Reconhecidos como campos internacionalizados, tradicionalmente representados em bases de dados internacionais e incorporando a publicação em inglês em títulos internacionais como padrão de publicação, os campos de Ciências Físicas, Tecnologia e Ciências da Vida \& Biomedicina revelaramse os campos de menor permanência da produção científica entre os títulos regionais indexados na WoS. Esse aspecto que demonstra a elevada dependência destes campos de títulos estrangeiros para a publicação de sua produção científica em um universo elitista como WoS. Padrão contrário ao comportamento exibido pelas Ciências Humanas e Sociais cuja indexação da produção científica não está condicionada à publicação em títulos estrangeiros, mas à inclusão dos títulos regionais na base.

Resultado similar foi observado por Mugnaini et al. (2019) sobre a produção de autores brasileiros observada na Plataforma Lattes, que identificaram forte predomínio da publicação em periódicos nacionais e regionais nos campos de Ciências Humanas, Linguística, Letras e Artes e Sociais, também relevante nos campos de Ciências Agrárias e da Saúde, embora nestes sejam identificados uma porção significativa de títulos internacionais. Nas Ciências Biológicas, Engenharias e Exatas a publicação ocorre predominantemente em periódicos estrangeiros, editados fora da América Latina e Caribe (MUGNAINI et al., 2019).

Por se constituir como um processo sociocognitivo, o desenvolvimento da atividade científica pode variar entre os locais em diferentes níveis permanência e evasão ao mesmo tempo (LEYDESDORFF; PERSSON, 2010) e, entre os diferentes campos do conhecimento em um mesmo local e ao mesmo tempo. Como é o caso de El Salvador que apresenta permanência de 84,62\% da produção em Ciências Sociais e Artes \& Humanidades e $100 \%$ de evasão nos demais campos. A mesma variação de níveis entre os campos ocorre na produção científica brasileira que apresenta permanência de $77 \% \mathrm{em}$ 
Artes \& Humanidades, 52\% em Ciências Sociais, 29,06\% em Ciências da Vida \& Biomedicina, 9,74\% em Tecnologia e 7,16\% em Ciências Físicas (Figura um), sinalizando diferentes taxas de evasão e permanência da produção científica e da presença dos periódicos dos países latinoamericanos e caribenhos entre os títulos mainstream nos diferentes campos.

Ciências da Vida \& Biomedicina concentra sua produção em quatro países, EUA $(31,85 \%)$, Brasil (18\%), Inglaterra (16,27\%) e Países Baixos (10,32\%), destino de 76,44\% dos artigos. Ciências Físicas e Tecnologia publicam a maior parte dos artigos em títulos de três países, EUA, Inglaterra e Países Baixos, respectivamente, 75,95\% e 78,94\% do total de artigos de cada campo (Tabela quatro), localidades que sediam os grupos editoriais comerciais com maior representação na WoS (GUÉDON, 2001, 2010, 2011; LARIVIERE; HAUSTEIN; MONGEON, 2015; PACKER 2011).

Artes \& Humanidades concentram 80,04\% dos artigos em títulos de cinco países, Brasil (36,66\%), Espanha (14,77\%), Chile (12,11\%), EUA (8,59\%) e Colômbia (7,91\%), enquanto as Ciências Sociais exibem um padrão mais disperso, uma vez que são seis os países que publicam 74,9\% dos artigos, Brasil (21,67\%), EUA (15,81\%), Inglaterra (14,43\%), Colômbia (9,36\%), Espanha (8,49\%) e México (5,13\%) (Tabela 4). Nesses campos a dependência internacional é menor e exibe maior equilíbrio ao incluir publicações regionais entre os destinos de suas publicações.

Neste estudo, entre os campos que exibem elevada dependência internacional, as maiores taxas de permanência nacional foram identificadas em: a) Ciências Físicas, no Brasil (7,16\%), México (6,51\%), Cuba (5,24\%), Chile (3,98\%); b) em Tecnologia, na Costa Rica (28,14\%), Colômbia (14,4\%), Venezuela (11,64\%), Brasil (9,74\%), Peru (7,05\%), Cuba (6,79\%) e México (4,58\%); e c) em Ciências da Vida \& Biomedicina, na Venezuela (31,14\%), Brasil (29,06\%), Cuba (18,57\%), Chile (17,14\%), Colômbia (12,44\%), Costa Rica (10,24\%), Paraguai $(9,62 \%)$ e Argentina (8,5\%) (Tabela 2). Resultados que expõe a internacionalização da produção científica destes campos nestes países e, a elevada dependência desses de títulos estrangeiros para ter sua produção representada entre a elite da produção científica mundial.

Entre os países que apresentam maior independência internacional para representação de sua produção científica na WoS estão: a) no campo de Artes \& Humanidades: El Salvador $(84,62 \%)$, Brasil (77\%), Colômbia (55,49\%) e Chile (51,62\%); e b) em Ciências Sociais: El Salvador (84,62\%), Paraguai (57,14\%), Cuba (57\%), Colômbia (54\%), Venezuela (54\%) e Brasil (52\%) (Tabela 2). Resultado que expressa algum sucesso na inclusão de títulos regionais entre as publicações indexadas pela base e, que pode representar um maior ou menor equilíbrio entre a representação da editoria regional entre a elite dos títulos mundiais e o intercâmbio científico global pela publicação em títulos estrangeiros. 
Em campos tradicionalmente internacionalizados, os mecanismos de avaliação da produção científica nacional nos países latino-americanos e caribenhos reproduzem os padrões de valoração globais, baseados na representação da produção científica em bases internacionais, dos quais o Qualis é um exemplo (CADERNOS DE SAUDE PUBLICA et al., 2019; RIZZOTTO; COSTA; LOBATO, 2019). A proposta de unificação do Qualis e sua estratificação pautada em indicadores fornecidos por bases de dados leva à manifestação de uma série de editores brasileiros acerca das diferenças entre os campos de produção do conhecimento, da possibilidade de fuga de artigos e de periódicos para outros campos que possuam melhor avaliação, levando ao subfinanciamento de títulos editados nos campos que não alcancem estratos superiores de avaliação (ASSOCIAÇÃO BRASILEIRA DE SAÚDE COLETIVA, 2019; FUNDAÇÃO OSWALDO CRUZ, 2019; RIZZOTTO; COSTA; LOBATO, 2019). Fato que se constitui como uma barreira à institucionalização dos veículos de comunicação na região, como pode ser observado na limitada permanência da produção científica em títulos latino-americanos e caribenhos, caso de 24,23\% dos artigos e, especialmente nos campos de Tecnologia e Ciências Físicas nos quais esse percentual é inferior a 10\% das publicações (Tabela 2).

Outro elemento associado à relação de poder centro-periferia exercida pela WoS aparece na extensão de sua influência nos campos que historicamente eram relegados pela base. Os títulos desses campos que tradicionalmente não possuíam indexação em bases internacionais, mas que possuem qualidade e o desejo de ampliar seu prestígio e visibilidade, passam a ser encarados como um potencial mercado de atuação para estes grupos editoriais uma vez que a relevância do FI e da indexação na WoS sejam incorporados entre os critérios de valoração da produção científica dessas áreas. E, sob essa perspectiva, precisa-se encarar a influência dos títulos indexados na WoS nos instrumentos de avaliação e a dependência dos campos do sistema de comunicação científica considerado mundial.

Em termos de estrutura editorial científica, os resultados obtidos por Mugnaini et al. (2019) apontam a relevância dos títulos brasileiros (30\%) e latino-americanos e caribenhos (7\%) para a publicação da produção científica nacional, responsáveis pela publicação de respectivamente $60 \%$ e 2\% dos artigos produzidos no Brasil incluídos na Plataforma Lattes. Um padrão diverso da produção científica brasileira representada entre os títulos mainstream, entre o qual somente $26,6 \%$ dos artigos é publicado nos títulos brasileiros (Tabela 2), embora estes sejam majoritários nos campos no quais existe os menores volumes de artigos, Artes \& Humanidades e Ciências Sociais (Figura 1).

Acerca da permanência na América Latina e Caribe, em todos os campos do conhecimento são os periódicos brasileiros o destino preferencial dos artigos produzidos na região $(15,21 \%)$ (Tabela 
3), resultado associado à proeminência da indexação dos títulos brasileiros em bases de dados internacionais em comparação aos demais países da região (RODRIGUES; ABADAL, 2014).

Ao observar os dados da Tabela 3 é possível perceber a dispersão da publicação regional concentrada em quatro países, EUA, Inglaterra, Brasil e Países Baixos, que juntos representam o destino de $75,1 \%$ dos artigos latino-americanos. Nesse contexto, a identificação de países anglo-americanos como destino dos artigos é consistente com as nacionalidades editoras predominantes entre os títulos considerados mainstream (GUEDÓN 2001, 2011; LARIVIERE; HAUSTEIN; MONGEON, 2015; PACKER 2011).

A presença dos títulos brasileiros como um dos destinos da produção científica regional, entre os títulos de nacionalidades que tradicionalmente dominam a indexação na WoS, evidencia seu destaque entre os países latinos na institucionalização dos periódicos científicos regionais. E tende a ser interpretado como parte de uma bem-sucedida integração ao sistema global de comunicação científica (VÉLEZ-CUARTAS; LUCIO-ARIAS; LEYDESDORFF, 2016). Essa preferência é um reflexodas ações para fortalecimento da editoria científica regional no país e, um indício do sucesso obtido por estas iniciativas, que abarcam desde a institucionalização dos Portais de Periódicos nas IES ao fortalecimento e atendimento aos critérios de inclusão na coleção SciELO.

Variável entre os campos do conhecimento, os destinos de publicação da produção científica latino-americana e caribenha apresentam distintas taxas de permanência e de evasão, como resultado do equilíbrio entre uma maior ou menor dependência do sistema global de comunicação científica. Um equilíbrio de difícil sustentação entre a) a internacionalização da produção científica regional, pela publicação em títulos mainstream estrangeiros, e b) a globalização dos periódicos científicos regionais, pela inclusão destes entre os títulos mainstream. Um aspecto sinalizando a constante participação no diálogo científico global e, uma maior dependência do sistema global de comunicação da Ciência, enquanto 0 outro evidencia a gradativa inclusão entre os títulos mainstream, o lento aceite entre a elite, e mesmo, uma maior independência e/ou isolamento do que é considerado Ciência Global, que tendem a diminuir à medida que sua posição entre o que é considerado central seja consolidada.

Estudos sobre a geografia da Ciência provêm uma base sobre a dinâmica de sua estrutura espacial (LEYDESDORFF; PERSSON, 2010) e, comumente são focados em análises acerca das localidades nas quais o conhecimento é produzido, representado pelo número de artigos publicados, em nível de países ou cidades, como meio de fornecer análises acerca da capacidade científica destes locais. Quanto à ênfase ao destino das publicações, estes costumam privilegiar análises da tipologia 
editorial e dos editores responsáveis pela publicação científica, em detrimento dos locais nos quais as contribuições acabam por ser publicadas. Este tipo de estudo possibilita a análise de aspectos associados ao destino, inclusive financeiro, do estoque de conhecimentos produzidos pela Ciência, 0 que implica em discussões sobre o acesso e a salvaguarda de seus documentos e a identificação de redes de edição científica e seu potencial de mercado.

Além disso, nestes estudos, a depender da fonte de dados, existe um maior ou menor viés geográfico que interfere na representação da produção científica mundial. Algo similar ocorre em estudos sobre rankings acadêmicos globais (JÖNS; HOYLER, 2013), sobre as metrópoles mundiais com a maior produção científica (CSOMÓS, 2018) e mesmo sobre o vínculo entre a citação e o local de produção do conhecimento (MAISONOBE et al., 2017), que raramente incluem a ciência produzida em países considerados periféricos e, que mais recentemente, quando apresentam alguma diversidade entre os centros científicos, esta se dá pela identificação de localidades emergentes entre os países centrais.

\section{CONCLUSÃO}

Em termos de estrutura editorial, os resultados apontam a importância dos títulos nacionais para a publicação da produção científica dos países da América Latina e Caribe, inclusive pela produção representada entre os títulos mainstream. Dado significativo da valoração e institucionalização de uma série de títulos nacionais, que obtém o reconhecimento internacional ao comporem os índices de basesde dados consideradas mais seletivas, mesmo que a representação dos campos ou dos países na base seja limitada ou pouco expressiva.

Publicar em títulos mainstream equivalha, para os pesquisadores e instituições, maximizar o prestígio obtido pelos resultados de seu trabalho, é também uma ação de acúmulo de capital científico. Editar esses títulos também é uma forma de acúmulo de capital, não só científico, mas financeiro, o que por si só confere poder e prestígio, mas é especialmente relevante em termos de estrutura do sistema de comunicação científica global, do acúmulo de vantagens e do poderio associado à esta posição. Nesse sentido, observa-se na América Latina e Caribe a necessidade, entre os diferentes campos do conhecimento, de alcançar o equilíbrio na representação de sua produção científica em títulos mainstream, entre aquilo que é publicado nos periódicos nacionais e o que é publicado em títulos estrangeiros. 
Percebe-se que os campos que possuem o maior volume de publicações são os campos de maior internacionalização, mas também os que exibem alguma dificuldade em incluir os títulos editados na região entre os que são considerados mainstream, embora a mesma barreira não ocorra com a publicação de seus artigos. Os campos em que há baixo volume de produção indexada na WoS, são os campos nos quais a maior parte da produção está publicada em títulos publicados na região. Embora variável entre os países, estes resultados apontam a necessidade dos campos internacionalizados de inclusão dos títulos editados na região entre os títulos mainstream e, nos campos nos quais cresce a representação de sua produção pela indexação dos títulos regionais, as Ciências Humanas e Sociais, a tendência em aderir aos padrões da base.

Esses resultados expressam a força das relações de dominação no sistema global de comunicação da Ciência, refletido nas avaliações nacionais da produção científica, na valorização e estímulo à publicação em periódicos estrangeiros, condicionando os pesquisadores da região a submissão de seus interesses de pesquisa e publicação ao que é aceito e valorado pelos editores de regiões centrais, controladas por editores comerciais.

\section{AGRADECIMENTOS}

Autora 1 teve bolsa de doutorado financiada pela CAPES código 001.

\section{REFERÊNCIAS}

ARAÚJO, Paulo Fernando Cidade de et al. Produtividade total dos fatores de produção na agricultura paulista e os investimentos públicos em capital humano. In: ARAÚJO, Paulo Fernando Cidade de; NICOLELLA, Alexandre Chibebe (Org.). Contribuição da FAPESP ao desenvolvimento da agricultura do estado de São Paulo. São Paulo: FAPESP, 2018. Cap.8, p. 357-396. Disponível em: http://fapesp. br/publicacoes/2018/agriculturasp.pdf. Acesso em: 28 nov. 2019.

ASSOCIAÇÃO BRASILEIRA DE SAÚDE COLETIVA. Critérios para classificação das revistas: apreciação da proposta de Qualis Periódico Referência. Notícias oficiais ABRASCO, 22 ago. 2019. Disponível em: https://www.abrasco.org.br/site/coordenadoresdepos/wp-content/uploads/ sites/2/2019/08/Carta-ABRASCO-sobre-Proposta-Qualis-Referencia.pdf. Acesso em: 08 jan. 2020.

BEIGEL, Fernanda. Publishing from the periphery: Structural heterogeneity and segmented circuits. The evaluation of scientific publications for tenure in Argentina's CONICET. Current Sociology, v.62, n.5, 2014. 
BEIGEL, Fernanda. El nuevo carácter de la dependencia intelectual. Cuestiones de Sociología, n. 14, jul. 2016.

BORNMANN, Lutz; WAGNER, Caroline; LEYDESDORFF, Loet. The geography of references in elite articles: Which countries contribute to the archives of knowledge? PLOS ONE, San Francisco, v. 13, n. 3, e0194805, 2018. DOI: https://doi.org/10.1371/journal.pone.0194805.

CADERNOS DE SAUDE PUBLICA et al. Contributions to the debate on the assessment of scientific research production. Cadernos de Saúde Pública, Rio de Janeiro, v. 35, n. 10, e00173219, 2019. DOI: http://dx.doi.org/10.1590/0102-311x00173219.

CSOMÓS, György. A spatial scientometric analysis of the publication output of cities worldwide. Journal of Informetrics, v. 12, i. 2, 2018, p. 547-566. DOI: https://doi.org/10.1016/j.joi.2018.05.003.

FUNDAÇÃO OSWALDO CRUZ. Carta aberta sobre a proposta do Qualis Periódico. FIOCRUZ, Rio de Janeiro, 2019. Disponível em: https://periodicos.fiocruz.br/sites/default/files/anexos/carta_FECF_ criterios-Qualis-Capes_v2_Fitosassina_22082019_0.pdf. Acesso em: 07 jan. 2020.

GUÉDON, Jean-Claude. Oldenburg's Long Shadow: Librarians, Research Scientists, Publishers, and the Control of Scientific Publishing. Association of Research Libraries. 2001. Disponível em: http:// www.arl.org/resources/pubs/mmproceedings/138guedon.shtml. Acesso em: 10 de jul. de 2011.

GUÉDON, Jean-Claude. Acesso Aberto e divisão entre ciência predominante e ciência periférica. In: FERREIRA, Sueli Mara Soares Pinto; TARGINO, Maria das Graças (Org.). Acessibilidade e visibilidade de revistas científicas eletrônicas. São Paulo: Editora SENAC: Cengage Learning, 2010. p. 21-79.

GUÉDON, Jean-Claude. El acceso abierto y La división entre ciencia "principal" y "periférica". Crítica y Emancipación, a. 3, n. 6, p. 135-180, 2011.

GUÉDON, Jean-Claude. Preface by Jean-Claude Guédon, chair on the expert group. In: EUROPEAN COMMISSION. Future of scholarly publishing and scholarly communication: report of the expert group to the European Commission. Luxembourg: Publications Office of the European Union, 2019.

JAGODZINSKI-SIGOGNEAU, M.; COURTIAL, Jean-Paul e LATOUR, Bruno. How to measure the degree of independence of a research system? Scientometrics, 1982. Disponível em: https://link.springer. com/article/10.1007/BF02018450. Acesso em: 23 dez. 2016.

JÖNS, Heike; HOYLER, Michael. Global geographies of higher education: the perspective of world university rankings. Geoforum, v. 46, may 2013. DOI: https://doi.org/10.1016/j. geoforum.2012.12.014

LARIVIÉRE, Vincent; HAUSTEIN, Stefanie; MONGEON, Philippe. The oligopoly of academic publishers in the digital era. PLOS One, v. 10, n. 6, 2015. Disponível em: http://www.plosone.org/article/ fetchObject.action?uri=info:doi/10.1371/journal.pone.0127502\&representation=PDF. Acesso em: 21 jun. 2016. 
LEYDESDORFF, Loet; PERSSON, Olle. Mapping the geography of science: Distribution patterns and networks of relations among cities and institutes. Journal of the American Society for Information Science and Technology, v. 61, i. 8, 2010. DOI: https://doi.org/10.1002/asi.21347.

LUCIO-ARIAS, Diana, VELEZ-CUARTAS, Gabriel, \& LEYDESDORFF, Loet (2015). SciELO citation index and web of science: Distinctions in the visibility of regional science. In Proceedings of the 15th Conference of the International Society for Scientometrics and Informetrics (ISSI), Istanbul, Turkey (pp. 1152-1160). Proceedings. https://www.researchgate.net/profile/Gabri el_Velez_Cuartas/ publication/281177604_SciELO_Citation_Index_and_Web_of_Science_Disti nctions_in_the_ Visibility_of_Regional/links/55da060708ae9d659491ea28.pdf

MAISONOBE, Marion et al. The global geography of scientific visibility: A deconcentration process (1999-2011). Scientometrics, i. 113, p. 479-493, 2017. Disponível em: https://link.springer.com/ article/10.1007/s11192-017-2463-2. Acesso em: 13 ago. 2019.

MIGUEL, Sandra. Revistas y producción científica de América Latina y el Caribe: su visibilidad en SciELO, RedALyC y SCOPUS. Revista Interamericana de Bibliotecología, Medellín, v. 34, n. 2, p. 187-199, 2011. Disponível em: http://www.scielo.org.co/scielo.php?pid=S0120$09762011000200006 \&$ script=sci_arttext. Acesso em: 29 fev. 2016.

MINNITI, Sergio; SANTORO, Valeria; BELLI, Simone. Mapping the development of open access in Latin America and Caribbean countries. An analysis of web of science core collection and SciELO citation index (2005-2017). Scientometrics, v. 117, n. 3, p. 1905-1930, 2018.

MUGNAINI, Rogério et al. Panorama da produção científica do Brasil além da indexação: uma análise exploratória da comunicação em periódicos TransInformação, Campinas, v. 3, e190033, 2019.

Disponível em: http://www.scielo.br/pdf/tinf/v31/2318-0889-tinf-31-e190033.pdf. Acesso em: 10 dez. 2019.

NEUBERT, Patricia; RODRIGUES, Rosângela. Dispersão dos artigos científicos latino-americanos indexados em bases de dados: um estudo da área de Artes \& Humanidades na Web of Science. ENANCIB In: ENCONTRO NACIONAL DE PESQUISA EM CIÊNCIA DA INFORMAÇÃO, 19., 2018, Londrina. Anais... Londrina: ANCIB, 2018.

PAASI, Anssi. Globalization, academic capitalism, and the uneven geographies of international journal publishing spaces. Environment and Planning A: Economy and space, v. 37, p. 769- 789, 2005. DOI: https://doi.org/10.1068/a3769

PACKER, Abel L. Os periódicos brasileiros e a comunicação da pesquisa nacional. Rev. USP, São Paulo, n. 89, maio 2011. Disponível em: http://rusp.scielo.br/scielo.php?script=sci_ arttext\&pid=S0103-99892011000200004\&lng=pt\&nrm=iso. Acesso em: 15 jun. 2016.

PEDROSA, Renato Hyuda de Luna; CHAIMOVICH, Hernan. Brasil. In: UNITED NATIONS EDUCATIONAL, SICENTIFIC AND CULTURAL ORGANIZATION. Relatório de Ciência da UNESCO: rumo a 2030: visão geral do cenário brasileiro. Paris: UNESCO; 2015.

PACKER, Abel; COP, Nicholas; SANTOS, Solange M. A Rede SciELO em perspectiva. In: PACKER, Abel et al. SciELO-15 Anos de Acesso Aberto: um estudo analítico sobre acesso aberto e comunicação científica. Paris: UNESCO, p. 41-66, 2014. 
RED DE INDICADORES DE CIENCIA Y TECNOLOGÍA - IBEROAMERICANA E INTERAMERICANA. Investigadores cada 1000 de la PEA (PF):2008-2017. Buenos Aires: RICYT, 2018. Disponível em: http://app.ricyt.org/ui/v3/comparative.html?indicator=CINVPEA\&start_year=2008\&end_year=2017. Acesso em: 08 abr. 2020.

RIZZOTTO, Maria Lucia Frizon; COSTA, Ana Maria; LOBATO, Lenaura de Vasconcelos Costa. The new Capes' criteria for the classification of journals and the repercussion in the field of public health. Saúde em Debate, Rio de Janeiro. v. 43, n. 122, 2019. DOI: https://doi.org/10.1590/01031104201912200

SANTIN, Dirce Maria; CAREGNATO, Sônia Elisa. The binomial center-periphery and the evaluation of science based on indicators. Investig. bibl, México, v. 33, n. 79, p. 13-33, jun. 2019 . Disponible en <http://www.scielo.org.mx/scielo.php?script=sci_arttext\&pid=S0187-358X2019000200013\&l ng=es\&nrm=iso $>$. accedido en 04 agosto 2020. Epub 08-Ene-2020. https://doi.org/10.22201/ iibi.24488321xe.2019.79.57930.

SOETE, Luc et al. Um mundo em busca de uma estratégia efetiva de crescimento. In: UNITED NATIONS EDUCATIONAL, SICENTIFIC AND CULTURAL ORGANIZATION. Relatório de Ciência da UNESCO: rumo a 2030: visão geral do cenário brasileiro. Paris: UNESCO; 2015.

STREHL, Letícia et al. Brazilian science between national and foreign journals: Methodology for analyzing the production and impact in emerging scientific communities. PloS one, v. 11, n. 5, p. e0155148, 2016. DOI: https://doi.org/10.1371/journal.pone.0155148

UNITED NATIONS EDUCATIONAL, SICENTIFIC AND CULTURAL ORGANIZATION. Science report: towards 2030. Paris: UNESCO; 2015.

TERRA-FIGARI, Lúcia I. Diseminación del conocimiento académico en América Latina. Montevideo. In: GORSKI, Sonnia Romero (Org.). Antropología social Y cultural en Uruguay 2007. Uruguay: UNESCO, 2008. p. 193-206.

VÉLEZ-CUARTAS, Gabriel; LUCIO-ARIAS, Diana; LEYDESDORFF, LOET. Regional and global science: publications from Latino America and Caribeean in the SciELO Citation Index and the Web of Science. El profesional de la información, v. 25, n. 1, ene./feb. 2016. 